\title{
Vacuum-Tube Acceleration Pickup
}

\author{
By Walter Ramberg
}

\begin{abstract}
The vacuum-tube acceleration pickup consists of a fixed indirectly heated cathode and two plates on either side of the cathode which are mounted elastically to deflect in response to acceleration normal to the plane of the plates. The tube has a natural frequency of about 800 cycles per second, and it has a flat response for sinusoidal accelerations from 0 to 200 cycles per second with enough output at accelerations of the order of $10 \mathrm{~g}$ to drive a recording galvanometer directly without the complications of an amplifier. The use of the tube is recommended in those applications in which its peculiar advantages of high natural frequency, ability to withstand large accelerations, and linearity outweigh the disadvantages of zero drift, warming-up time, high current consumption, and the need of filtering to remove natural frequency response.
\end{abstract}

\section{Introduction}

The vacuum-tube acceleration pickup described in this paper represents the most recent stage in a development which has been carried on for the Bureau of Aeronautics, Navy Department, at the National Bureau of Standards and at Sylvania Electric Products, Inc., since 1941.

The purpose of the investigation was to develop an acceleration pickup that would have a flat response for sinusoidal accelerations with frequencies up to $300 \mathrm{c} / \mathrm{s}$ and that would have enough output at accelerations of the order of $10 \mathrm{~g}$ to drive a recording galvanometer directly without the complication of an amplifier. It was thought from the beginning that this might be accomplished with a vacuum tube in which acceleration causes electrodes to deflect elastically relative to each other.

The relative deflection of electrodes in a vacuum tube had been used as early as 1936 by Ross Gunn of the Naval Research Laboratory ${ }^{1}$ for measuring small displacements. A more recent application

1 Ross Gunn, Apparatus for the detection of displacements, U. S. Patent 2,155,420. Reissued Feb. 27, 1940, Re. 21,372. is the microphonic electron tube for measuring small changes in pressure, which was proposed by Jerome Rothstein in $1943 .^{2}$

2 Jerome Rothstein, Microphonic electron tube, U. S. Patent 2,389,935 (Nov. 27, 1945).

\section{Contents}

Page

I. Introduction

II. Characteristics.

1. Construction _... 392

2. Frequency _. _... 392

3. Resistance _. _ _ _ _ _ _ _ 392

4. Calibration factor _...

5. Response to transverse acceleration

6. Wave form

7. Stability - .

8. Range of linear response _._._._._. 394

III. Method of using pickup _..._. 394

IV. Field of application

V. Conclusion

VI. Appendix

Vacuum-Tube Acceleration Pickup $\quad 391$ 


\section{Characteristics}

\section{Construction}

The vacuum-tube acceleration pickup is a twin diode consisting of a fixed indirectly-heated cathode and two plates, one to either side of the cathode. The plates are elastically mounted to deflect in response to acceleration normal to the plane of the plates.

Figure 1 shows the arrangement and mounting of electrodes in a vacuum tube of a type of which an experimental lot of 95 was built by Sylvania Electric Products, Inc. as type SD 759. The cathode, $C$, is anchored in the two mica plates, $M$. The mica plates, $M$, are connected into a rigid box by transverse bars $B_{1}, B_{2}, B_{3}, B_{4}$. The box is fastened to the base of the tube by vertical pins, $B_{5}, B_{6}, B_{7}, B_{8}$. The two anode plates, $P_{1}, P_{2}$, consist of thin sheet metal wrapped into a closed rectangular section; this reduces the mass of the plates to a low value, thereby reducing the warming-up time of the electrodes. The plates are connected to two relatively slender vertical rods, $R_{1}, R_{2}$, and $R_{3}, R_{4}$, respectively. These rods are welded to transverse bars $B_{3}, B_{4}$. The plate leads are brought from the transverse bars $B_{3}, B_{4}$, to the vertical pins, $B_{9}, B_{10}, B_{11}, B_{12}$, by two heavy connectors each.

The fundamental frequency of the plate structure for bending normal to the plane of the plate was computed by treating the structure as an elastic system with a concentrated mass at the center of gravity of the plate and distributed masses in the supporting wires, which are subjected to flexure and torsion by the vibration of the plate normal to its plane. The diameters and lengths of the wires were computed to give a fundamental frequency in this mode of vibration of the order of $750 \mathrm{c} / \mathrm{s}$. The natural frequencies of vibration in other modes were estimated to be much higher than $750 \mathrm{c} / \mathrm{s}$.

Figure 1 (c) shows the complete pickup. It is housed in a T-9 glass envelope approximately 2 inches long and has a loctal base.

\section{Frequency}

The lowest natural frequency was determined experimentally by connecting the pickup into the Wheatstone bridge circuit of figure 2 with a cathode-ray oscilloscope between $C$ and $D$, tapping the pickup with a pencil or a finger, and matching the output on the oscilloscope by the output of known frequency for an audio oscillator.

The natural frequency for the 95 pickups of this design that have been built was found to be

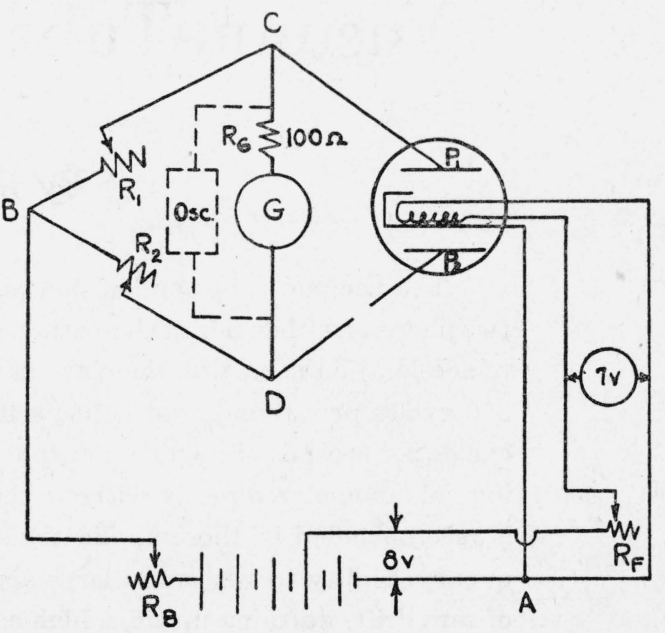

FIGURE 2.-Wheatstone-bridge circuit for measuring and recording output of tube.

between 595 and $915 \mathrm{c} / \mathrm{s}$, with an average value of $847 \mathrm{c} / \mathrm{s}$.

\section{Resistance}

The resistances $R_{P 1}, R_{P 2}$ from each plate to the cathode were measured with the Wheatstone bridge of figure 2 after a warming-up time of several minutes. The heater voltage was taken as 7 volts and the plate voltage as 10 volts. These are the normal operating voltages for which the tube had been designed. The bridge was balanced by adjusting $R_{B}, R_{1}, R_{2}$ until the potential difference between $A$ and $B$, as measured with a voltmeter, was 20 volts, and that between $A$ and $C$, and $A$ and $D$ was 10 volts. Under this condition of balance, $R_{1}, R_{2}$ must be equal to $R_{P 1}, R_{P 2}$, respectively.

The resistances $R_{P 1}, R_{P 2}$ for the 95 tubes ranged from 88 to 314 ohms, with an average value of $157 \mathrm{ohms}$. The ratio of the larger one of the two resistances to the smaller one was from 1.00 to 1.46 , with an average value of 1.15 .

The resistance of the heater at 7 volts was of the order of $14 \mathrm{ohms}$. The total power consumption in the circuit of figure 2 is, therefore, of the order of 7 watts. 


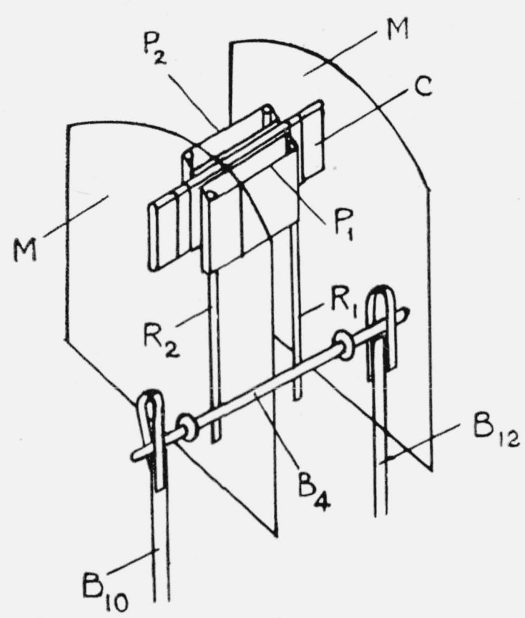

(a)

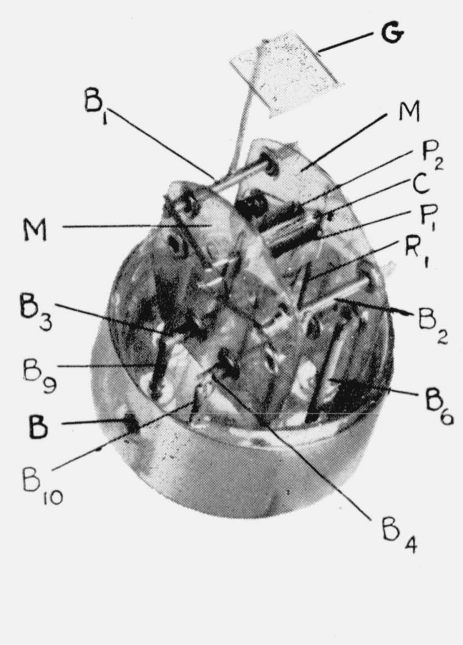

(b)

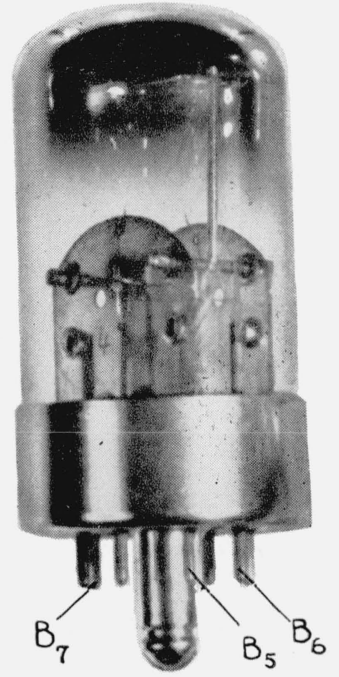

(C)

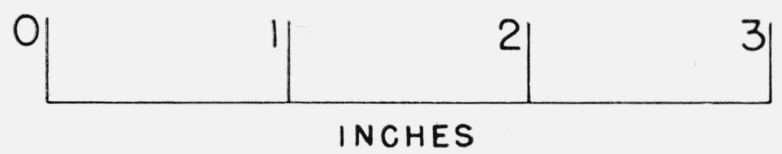

FigURE 1.-Arrangement of electrodes in tube. 


\section{Calibration Factor}

The calibration factor was computed from the output corresponding to a change in acceleration $2 g$ on the assumption of direct proportionality between acceleration and relative change in plate-cathode resistance. This assumption has been verified experimentally for accelerations up to $45 \mathrm{~g}$. The tube was connected into the Wheatstone-bridge circuit, figure 2 , which was roughly balanced by making the resistances $R_{1}$, $R_{2}$ equal to the previously measured plate-cathode resistances $R_{P 1}, R_{P 2}$. The resistances $R_{1}, R_{2}$ were adjusted further, keeping their sum constant until the pointer on galvanometer $G$ was near the middle of the scale. The tube was allowed to warm up for 15 minutes, and was then slowly rotated with its axis horizontal through 360 degrees, and the maximum and minimum currents were noted on a galvanometer, $G$, with an impedance of $100 \mathrm{ohms}$ between $C$ and $D$. The maximum unbalance current corresponded to $+1 g$ on one plate (in a horizontal position on top) and the minimum current corresponded to $-1 g$ on the same plate (in a horizontal position on the bottom). The difference in unbalance current $I_{2 g}$ therefore corresponded to a change in acceleration $2 g$. The calibration factor, $K$, was computed from the following equation, derived in the appendix:

$$
K=\frac{\Delta R}{R} / \frac{a}{g}=\left(5+\frac{R_{P 1}+R_{P 2}}{40}\right) I_{2 g},
$$

where $\frac{\Delta R}{R}=\frac{1}{2}\left(\frac{\Delta R_{P 1}}{R_{P 1}}+\frac{\Delta R_{P 2}}{R_{P 2}}\right)=$ average relative

change in plate-cathode resistance corresponding to acceleration, $a$, normal to the plate.

The values of $K$ for the 95 tubes ranged from 0.0029 to 0.0072 , with an average value $K=0.0045$. This corresponds to an output of the order of 0.2 $\mathrm{ma} / \mathrm{g}$. The sensitivity of the tube, as measured by the calibration factor $K$, depends on the plate voltage and on the heater voltage. Tests have indicated that $K$ may be increased about 40 percent by increasing the plate voltage from 10 to 12 volts, keeping the heater voltage at a constant value between 6.5 and 7.0 volts.

\section{Response to Transverse Acceleration}

The response to transverse acceleration was measured on a shaking table vibrating sinusoidally at $32 \mathrm{c} / \mathrm{s}$. with a displacement amplitude of about 0.05 inch, producing an acceleration amplitude of about $5 g$. The tube was first placed with the acceleration normal to the plates and then with the acceleration parallel to the plates and normal to the axis of the tube. The transverse response was measured by the ratio of amplitudes on a cathode-ray oscilloscope, with the tube in the second and in the first position.

The transverse response was found to be negligible for the first batch of six tubes that was tested. Tests on the remaining tubes were omitted as superfluous.

\section{Wave Form}

The wave form was observed on a cathode-ray oscilloscope when the tube was mounted on the end of a cantilever beam, which was vibrated in its fundamental mode as a free elastic body. There was no visible deviation from a sinusoidal shape for each one of the 10 tubes tested for wave form.

\section{Stability}

The zero drift was measured by connecting the tube into the Wheatstone-bridge circuit of figure 2 and recording the change in unbalance current with time without subjecting the tube to any change in acceleration. The measured change in unbalance current was divided by the change in current $I_{2 g} / 2$, corresponding to an acceleration of $1 g$, in order to express the drift as a "drift in $g$. ."

The zero drift in 15 minutes, after a warming-up period of 15 minutes, exceeded $1 g$ for 30 of the 95 tubes; it was less than $0.2 g$ for 25 tubes; it was too small to be measured for 7 tubes.

Large drifts could be traced sometimes to temperature changes around the tube, to fluctuations in heater voltage, or to poor electric contact in the connections to the base of the tube. The drift could be reduced in such cases by enclosing the tube in a small box, by controlling the heater voltage, or by soldering the leads from the bridge to the pins projecting from the base of the tube. However, an unpredictable drift of the order of $\pm \mathrm{l} g$ in a few minutes took place from time to time in certain tubes, even after all these precautions were taken. Fortunately, the drift tends to decrease with the age of the tube. Twentynine tubes that showed drifts in excess of $1 g$ were 
retested after 6 months of storage. Of these tubes, none had drifts exceeding $1 g, 12$ had drifts from $0.2 g$ to $1.0 g$, and 17 had drifts below $0.2 g$.

Some measurements of zero drift were made at a plate voltage of 12 volts and a heater voltage of 6.5 volts, which, as mentioned above, may lead to an appreciable increase in sensitivity. The drift at these voltages was either less than or equal to that measured at the voltages spcified in figure 2.

\section{Range of Linear Response}

The tubes were originally designed for a maximum acceleration of $20 \mathrm{~g}$. However, experience has indicated that they will function under much higher accelerations.

One tube was calibrated at accelerations up to $45 \mathrm{~g}$ by attaching the tube to a shaking table, which was run at constant amplitude of about 0.19 inch up to a speed of about $50 \mathrm{c} / \mathrm{s}$. The tube was connected into the Wheatstone bridge of figure 2, and the unbalance current of the bridge was fed into a type $C$ galvanometer (internal resistance, $9 \mathrm{ohms}$; frequency, $430 \mathrm{c} / \mathrm{s}$; d-c sensitivity, $2 \mathrm{~mm} / \mathrm{ma}$ ) in a Heiland six-channel oscillograph. The unbalance current was recorded as the shaking table was allowed to coast down after switching off the current to the driving motor. It was found that the unbalance current was a linear function of the acceleration over the whole range of the measurements. At a later date the same tube was subjected to an acceleration of $117 \mathrm{~g}$ on the same shaking table; its output was found to correspond to the same calibration factor as that obtained on the calibration to $45 \mathrm{~g}$.

Other tubes were frequently subjected to accelerations well above $20 \mathrm{~g}$ without showing a change in calibration factor. However, severe and prolonged shaking was in several instances accompanied by a shift in the "zero."

\section{Method of Using Pick-up}

The method of using the vacuum-tube acceleration pickup is most readily illustrated with a specific example.

Figure 3 shows a tube in use for recording vertical accelerations at the center section of a model "airplane" subjected to a drop test.

The tube is connected into the Wheatstone bridge of figure 2, which is built into the control panel of figure 4. This control panel is equipped with a meter, $M$, which may be switched in to indicate heater voltage, plate voltage, or unbalance current.

Connections to the pins projecting from the base of the tube were made by soldering on leads of No. 24 insulated copper wire. The soldering was done carefully to avoid damage to the glass seal around the pins by overheating. Solder connections were used in preference to connections through a standard socket to eliminate errors from changes in contact resistance between pins and sleeves in the socket. Parallel pairs of wires were used in each heater lead to prevent excessive heating and to retain the flexibility of the No. 24 wire:

Special care was taken to clamp the tube to the structure in such a manner that it would experience the same acceleration as the structure. For this purpose the tube was clamped at the metal sleeve in a split clamp of $1 / 32$-inch brass tubing, which is shown in figure 5. A layer of transparent scotch tape was wrapped on the metal sleeve of the tube before inserting in the clamp to distribute the clamping force, thereby preventing breakage of the glass envelope during clamping.

The tube was then allowed to heat up at least 15 minutes to attain equilibrium. A type $C$ galvanometer in a Heiland six-channel oscillograph was connected in at $G$ in figure 2 with suitable resistance in series or in parallel to limit the sensitivity to a safe value. The resistor $R_{1}$ was adjusted while gradually increasing the sensitivity until the galvanometer indicated balance. The resistor $R_{F}$ in the heater circuit was then adjusted until the meter, $M$, in the control panel, figure 4, indicated 7 volts. The resistor $R_{B}$ in series with the bridge was next adjusted until the meter indicated 10 volts.

The balance of the bridge was again checked and, if necessary, adjusted.

The recorded output of the tube was calibrated by subjecting the tube to a change in acceleration $2 g$ and recording the change in output. For this purpose the plates were first placed horizontally with button B, figure 1 , at " 9 o'clock" and the output was recorded. Then the tube was rotated about its horizontal axis to place the button at "3 o'clock" and the output was recorded again. 


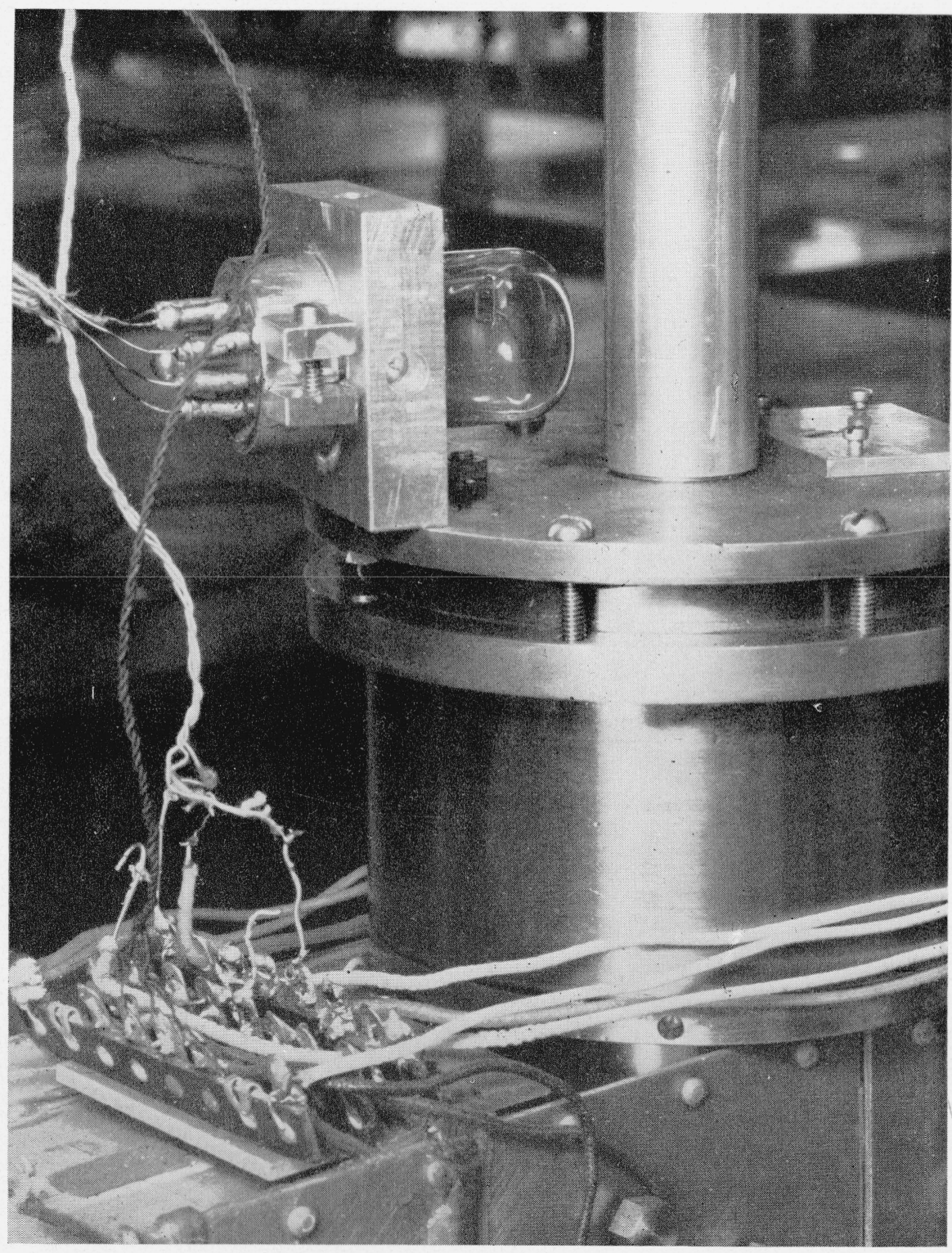

Figure 3.-Tube mounted for recording vertical acceleration at center section of model "airplane". 


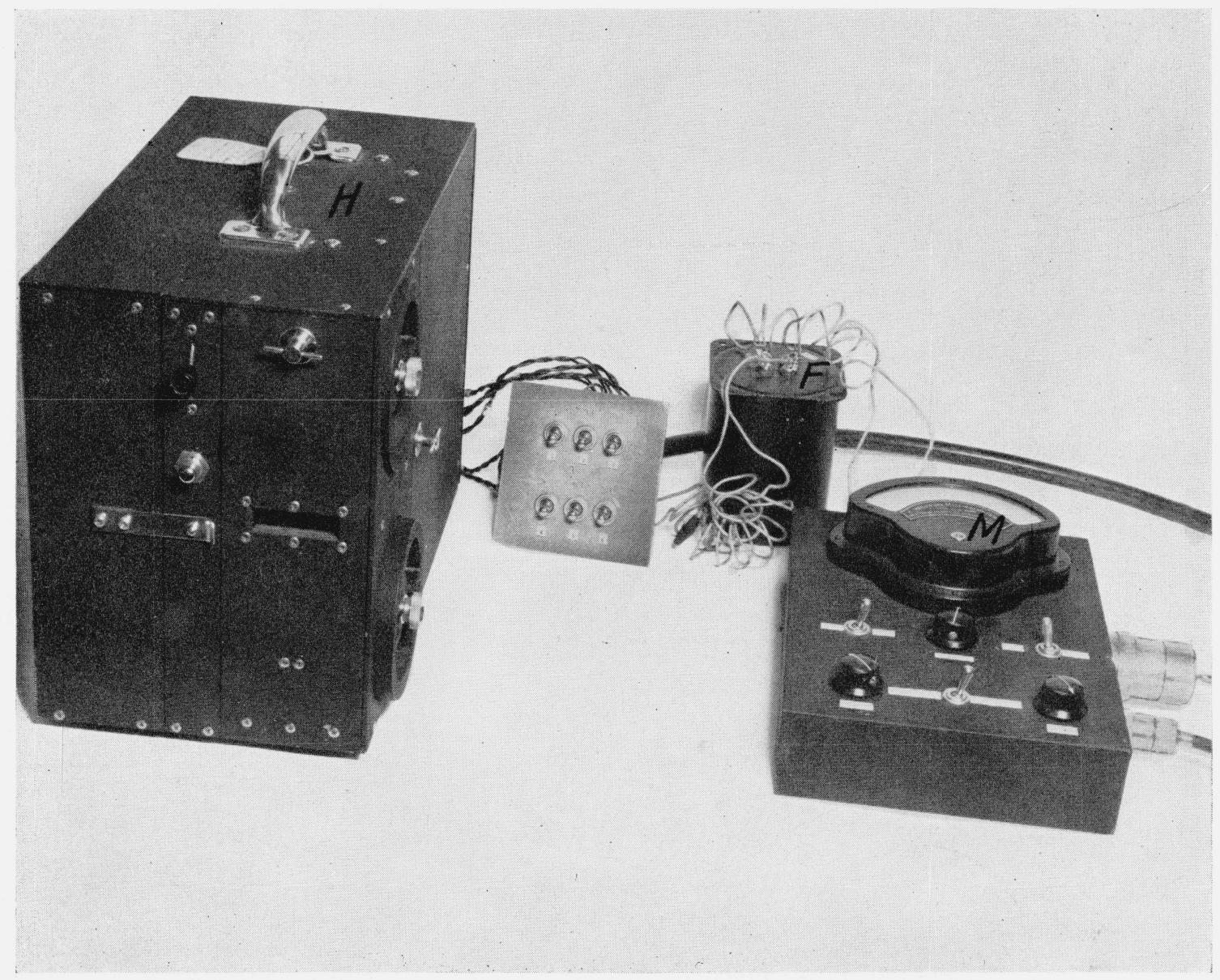

Figure 4.-Recorder, filter, and control panel for tube. 


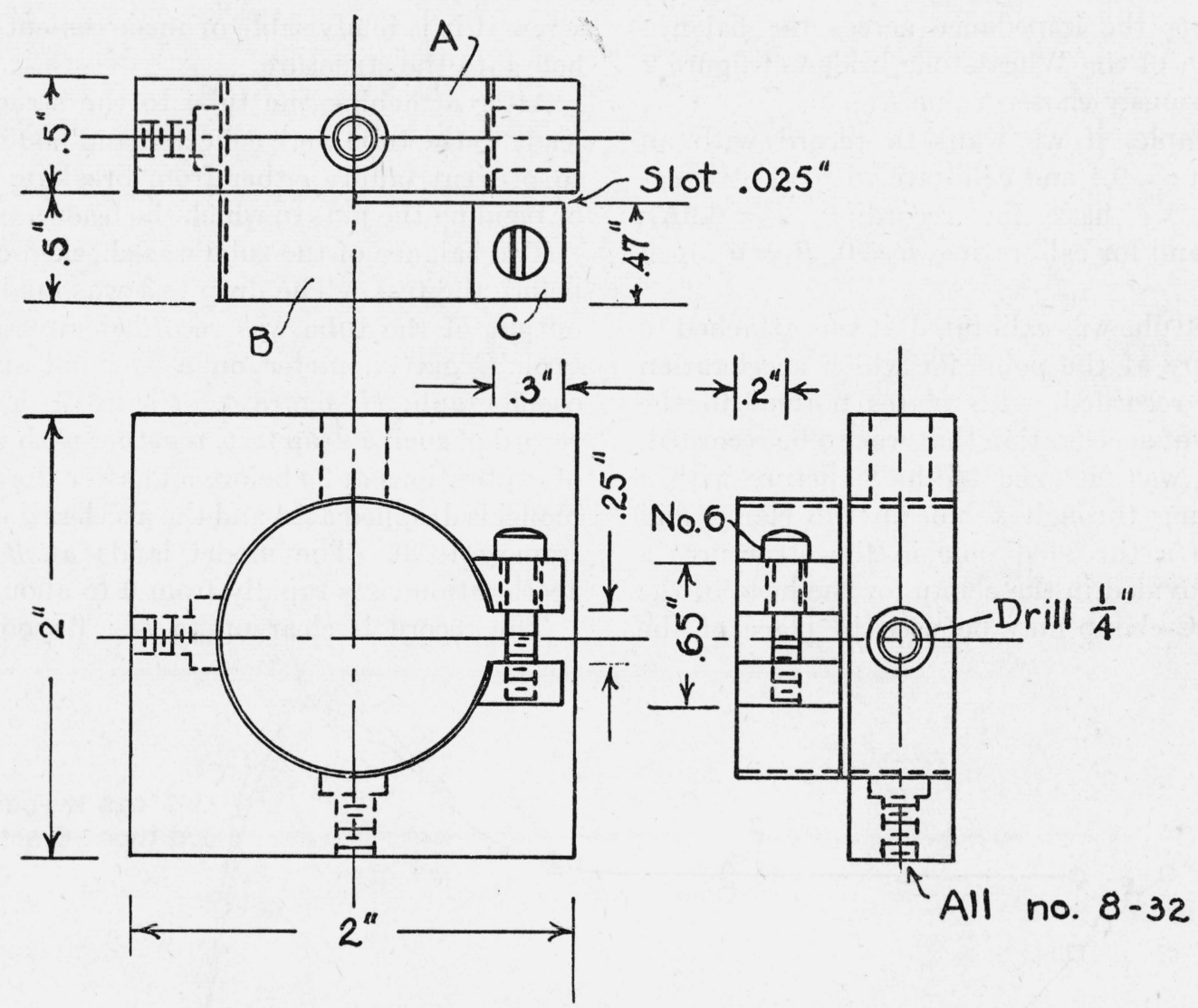

Figure 5.-Split clamp for holding tube.

$A$, Aluminum alloy; $B, 1 \frac{1}{4}$-in. by $1 / 32$-in. brass tube, press fit in $A ; C$, brass lugs, silver solder to $B$.

Rotation through 180 degrees is particularly simple when the tube is held in the square-edge clamp of figure 5. Rotation with the clamp has the additional important advantage of preventing errors in indicated output caused by changes in physical contact between the tube and its surroundings.

In some applications in which the accelerations to be recorded are much larger than $2 g$, it is necessary to calibrate with a larger output on the recording paper.

The larger output may be obtained either directly by imposing larger accelerations or indirectly by increasing the sensitivity (lowering the attenuation) for the calibration at $2 g$ by a known factor.

A portable calibrator has been developed at the National Bureau of Standards for applying known accelerations up to $20 g$ to four vacuum-tube acceleration pickups simultaneously.

Figure 6 shows a simple circuit for changing the sensitivity, or attenuation, by a factor $\alpha$ with-

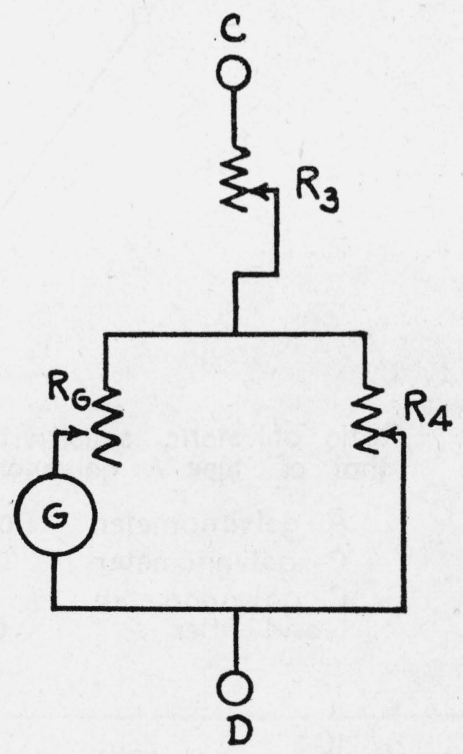

FIGURE 6.-Circuit for changing sensitivity of tube without changing input impedance into recorder.

$\alpha=$ attenuation; $R_{3}=(1-\alpha) R_{G} ; R_{4}=\frac{\alpha}{1-\alpha} R_{G}$. 
out changing the impedance across the balance points $C, D$, of the Wheatstone bridge of figure 2 from a previously chosen value $R_{G}$.

For example, if we want to record with an attenuation $\alpha=0.1$ and calibrate with an attenuation $\alpha=1$, we have for recording, $R_{3}=0.9 R_{g}$, $R_{4}=R_{G} / 9$; and for calibrating, $R_{3}=0, R_{4}=\infty$ (open circuit).

After the tube was calibrated, it was attached to the structure at the point for which acceleration was to be recorded, with plates normal to the component of acceleration that was to be recorded. The clamp was fastened to the structure with a screw passing through a hole in the clamp and fitting into a threaded hole in the structure; a recess is provided in the clamp for the head of the screw. A C-clamp may be used in place of the screw if it is inadvisable or inconvenient to drill a hole into the structure.

After attaching the tube to the structure, the leads to the tube were collected and fastened down to prevent failure either from breaking the leads or bending the pins to which the leads are soldered.

The balance of the tube was checked once more before the test. The drop test was made and the output of the tube was recorded directly with a type $C$ galvanometer on a Heiland six-channel oscillograph, $H$, figure 4 . Figure 7 (a) shows a record of such a drop test, together with the record of calibrations at $2 g$ before and after the test. The model is dropped at $A$ and the acceleration changes from $g$ to 0 . The model lands at $B$ and the acceleration rises rapidly from 0 to about $4 g$ at $C$.

The record is clear up to $D$. Beyond $D$ it is

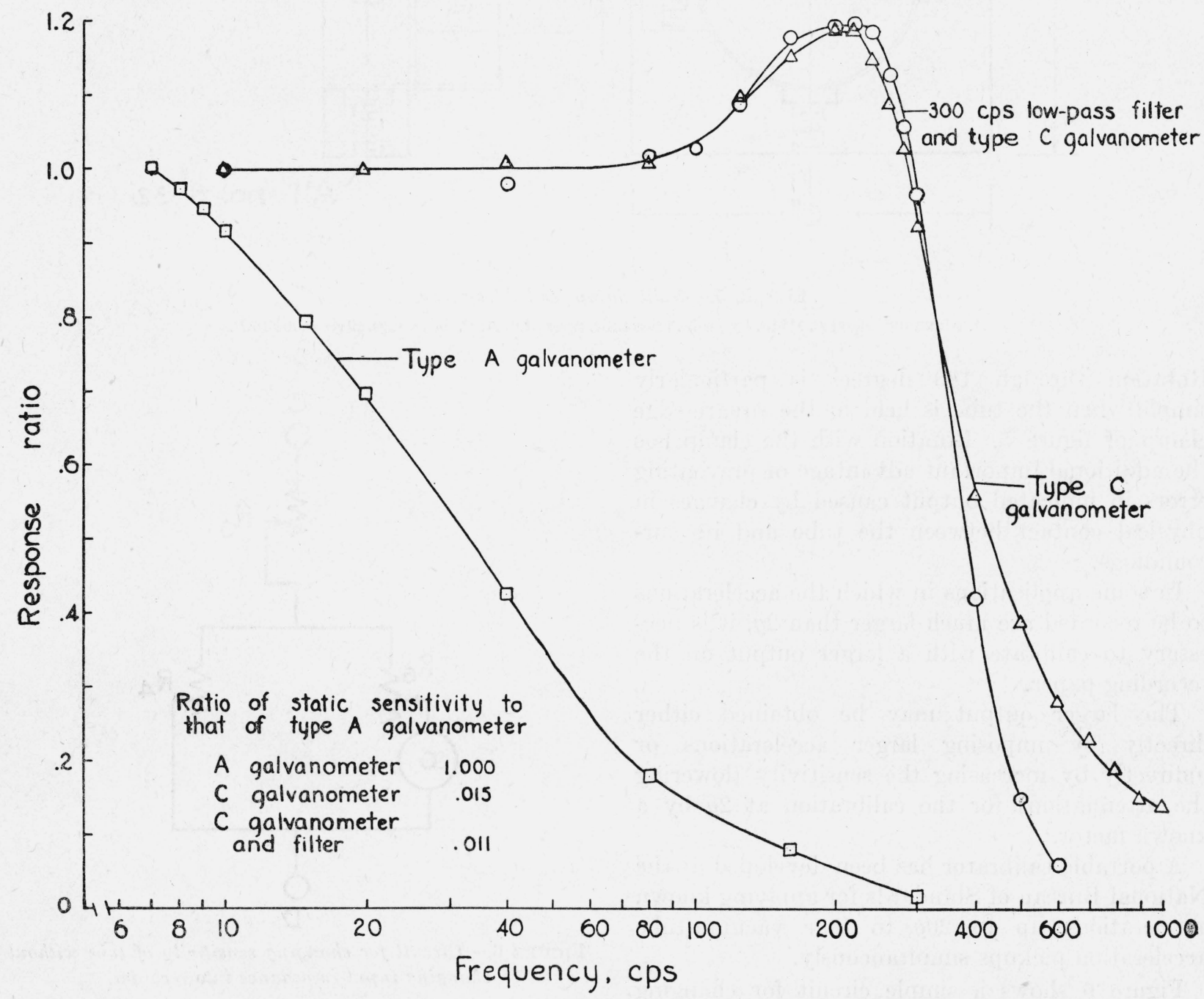

FIGURe 8.-Response ratios for galvanometers and filter when used with Wheatstone-bridge circuit in figure 2. 


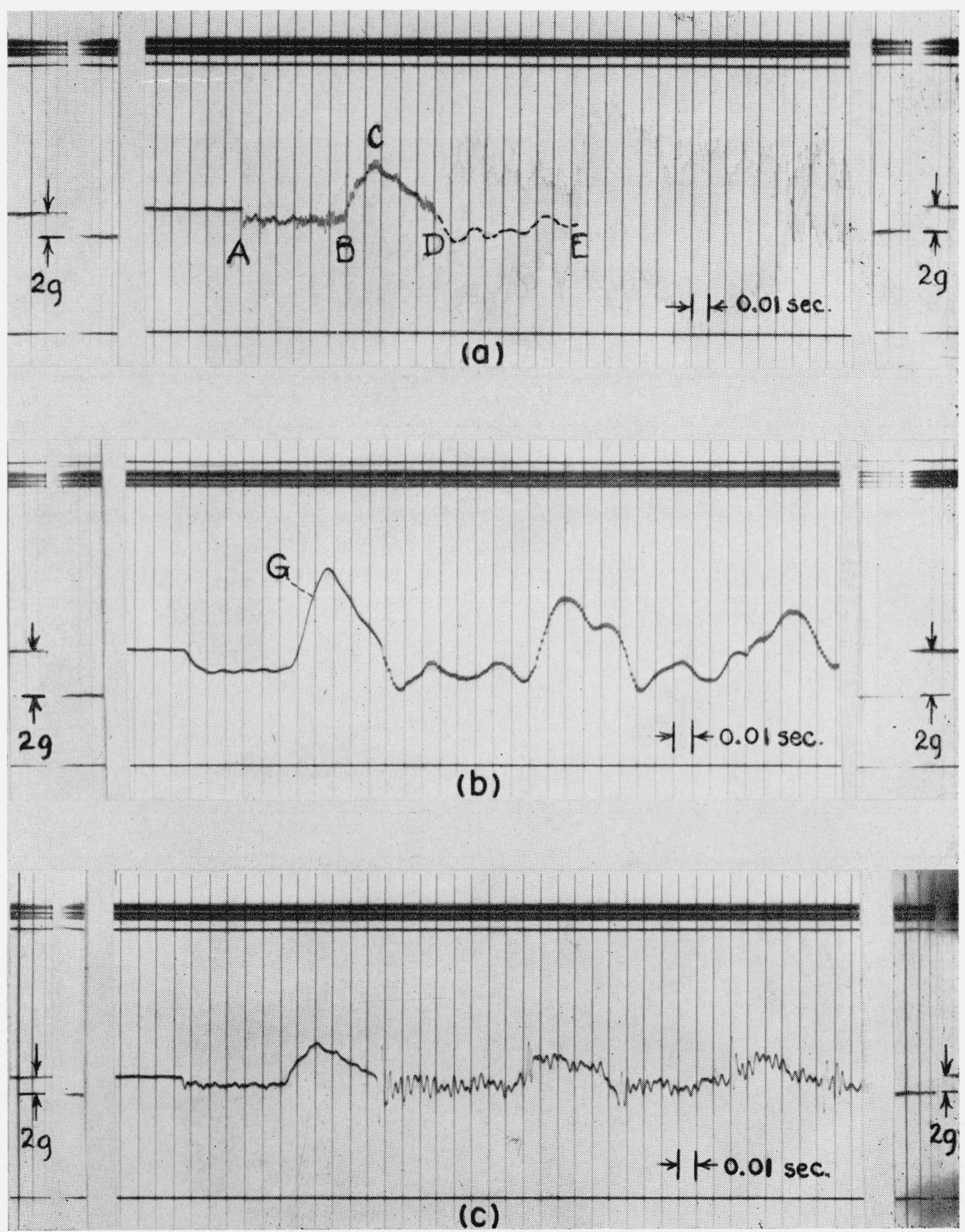

FIGURE 7.-Records of identical drop tests with high-frequency galvanometer type C without filter, low-frequency galvanometer type $A$ without filter, and high-frequency galvanometer type $C$ with filter.

(a) Type C, no filter; (b) type A, no filter; (c) type C, UTC filter. 
marred by superimposed oscillations at the natural frequency of the plates in the tube (about $800 \mathrm{c} / \mathrm{s}$ ), which are too faint to be clearly seen in figure 7 (a). The acceleration can be estimated, nevertheless, as long as the superimposed oscillation is clearly recorded, by connecting the mean points of the oscillation. This is indicated by the dotted curve in figure 7 (a). But even this expedient fails beyond $E$, where the oscillations become of too large amplitude to be recorded.

Satisfactory records can be obtained under these conditions by reducing the recorded output at the natural frequency of the tube. This may be accomplished either by using a recording galvanometer, which is more efficient in attenuating vibrations at the natural frequency of the tube, or by inserting a suitable electric filter.

The response ratio (ratio of sensitivity to d-c sensitivity) of the type $C$ galvanometer at the natural frequency of the tube $(800 \mathrm{c} / \mathrm{s})$ is about 0.19 according to figure 8 ; this is apparently insufficient to reduce natural frequency response to an acceptable value.

Figure 7 (b) shows a record obtained with a Heiland type $A$ galvanometer which has a natural frequency of $40 \mathrm{c} / \mathrm{s}$ as compared to $430 \mathrm{c} / \mathrm{s}$ for the type $C$ galvanometer. The response ratio at $800 \mathrm{c} / \mathrm{s}$ is less than 0.01 , according to figure 8 . The output of the tube was attenuated to 0.029 of its former value in order to compensate for the increased sensitivity of the type $A$ galvanometer as compared to the type $C$ galvanometer. The record is much easier to follow than that of figure 7 (a); however, it is unsatisfactory insofar as the galvanometer is unable to follow rapidly varying accelerations, as at $G$, because of its relatively low natural frequency.

A satisfactory record without appreciable response at the natural frequency may be obtained by inserting a low-pass filter, $F$, figure 4 , between the bridge and the galvanometer. Figure 8 shows the response curve for a type $C$ galvanometer with a filter (UTC filter 93051) built by the United Transformer Co., which has proved satisfactory for this purpose. This filter is practically flat up to $300 \mathrm{c} / \mathrm{s}$ and cuts off sharply to a relative response of less than 0.01 at $800 \mathrm{c} / \mathrm{s}$. The weight of the filter is less than 3 pounds.

Figure 7 (c) shows a record of the drop test with the filter placed ahead of the galvanometer, as shown in figure 4 . The record gives the same accelerations as figure 7 (a) and the response at the natural frequency has been suppressed. The vibrations with a frequency of about $250 \mathrm{c} / \mathrm{s}$ in the right-hand portion of the record were ascribed to elastic oscillations of a portion of the model "airplane" excited by the rebound from the landing impact. Insertion of the filter has resulted in a loss of current sensitivity of about 30 percent.

\section{Field of Application}

The use of the vacuum tube is recommended in those applications in which its peculiar advantages of high output, high natural frequency, high capacity, and linearity outweigh the disadvantages of zero drift, warming-up time, high-power consumption (about 7 watts for tube and Wheatstone bridge of fig. 2), and the need of filtering to remove natural frequency response.

A potential advantage of the vacuum tube acceleration pickup over other acceleration pickups is the possibility of building it at relatively low cost in large quantities using the special technics developed by the manufacturers of vacuum tubes.

The high output and high natural frequency of the vacuum tube makes it possible to record rapidly varying accelerations without requiring an amplifier. This is a desirable feature in flight tests of small airplanes where space and weight are at a premium. It also simplifies tests in the laboratory when amplifying channels are not readily available.

The high capacity of the pickup is advantageous in recording accelerations over a wide range and in preventing damage from sudden high accelerations.

The linearity of the pickup makes possible the filtering out of high-frequency response, regardless of amplitude to produce a record proportional to acceleration.

The zero drift found in most of the tubes makes them unsuitable in those flight tests in which the imposed acceleration is unknown over periods of time measured in minutes. The zero drift is not serious in recording either very large accelerations or in recording changes in acceleration over a period of time measured in seconds. Typical 
illustrations of the latter are drop tests, and impact tests in which the impact is softened by cushioning, so that it can be recorded with a pickup having a flat response up to $300 \mathrm{c} / \mathrm{s}$.

The warming-up time of 15 minutes represents a drain on the available power, which may be serious in certain applications. It also introduces an undesirable delay in adjusting the equipment.

The filter represents an added item of expense (about $\$ 40.00$ ) and of weight (about $3 \mathrm{lb}$ ), which may be avoided by using pickups of other types with internal damping.

The vacuum-tube acceleration pickup is being tried out under various service conditions by several flight research laboratories to indicate its field of usefulness more specifically. Service experience may also indicate certain improvements in design and construction that would overcome some of the disadvantages of using a vacuum tube to measure accelerations.

\section{Conclusion}

The vacuum tube acceleration pickup appears to be a convenient means for measuring accelerations in the range from $5 \mathrm{~g}$ to $40 \mathrm{~g}$. It has an advantage over other acceleration pickups in combining high sensitivity with high natural frequency thereby making it possible to record rapidly varying accelerations directly without the complication of an intermediate amplifier. It has a disadvantage over other pickups in being more subject to zero drift, in requiring a warming-up time of about 15 minutes to reach equilibrium, and in requiring a filter to remove response at its natural frequency.

\section{Derivation of equation 1}

The proof of equation 1 follows from the expression for the current $i_{G}$ flowing through the galvanometer resistance $R_{G}$ when the Wheatstone bridge of figure 9 is unbalanced by changing two of the resistances by $\Delta R_{1},-\Delta R_{2}$ as indicated. This current, according to page 45 of reference, ${ }^{3}$ is:

In our case

$$
\begin{gathered}
i_{G}=\frac{E\left[\left(R_{1}+\Delta R_{1}\right) R_{2}-R_{1}\left(R_{2}-\Delta R_{2}\right)\right]}{R_{G} 2 R_{1} 2 R_{2}+2 R_{1}^{2} R_{2}+2 R_{1} R_{2}{ }^{2}} \\
=\frac{E}{4 R_{G}+2\left(R_{1}+R_{2}\right)}\left(\frac{\triangle R_{1}}{R_{1}}+\frac{\Delta R_{2}}{R_{2}}\right) .
\end{gathered}
$$

$$
\begin{gathered}
i_{G}=I_{2 g} \\
\left(\frac{\Delta R_{1}}{R_{1}}+\frac{\Delta R_{2}}{R_{2}}\right)=\left(\frac{\Delta R_{P 1}}{R_{P 1}}+\frac{\Delta R_{P 2}}{R_{P 2}}\right)=\frac{2 \Delta R}{R},
\end{gathered}
$$

where $\Delta R_{P 1}, \Delta R_{P 2}$ are the changes in plate-cathode resistance corresponding to a change in acceleration $2 g$. Substituting equation 3 in equation 2 and solving for $\Delta R / R$ gives

$$
\frac{\Delta R}{R}=\left(2 R_{G}+R_{1}+R_{2}\right) \frac{I_{2 g}}{E}
$$

In the case of figure 2

$$
R_{G}=100 \mathrm{ohms}, E=20 \text { volts. }
$$

Inserting equation 5 in equation 4 , and remembering the definitions of calibration factor $K$, gives equation 1 :

$$
K=\frac{\Delta R}{R} / \bar{a}=\frac{\Delta R}{R} / 2=\left(5+\frac{R_{1}+R_{2}}{40}\right) I_{2 g} .
$$

Washington, July 19, 1946.
Further experience in the use of the vacuumtube acceleration pickup in flight tests as well as in the laboratory will be necessary to indicate its full scope of usefulness.

The author takes this opportunity to thank the Bureau of Aeronautics for permission to publish this material. He also acknowledges his debt to the engineers of Sylvania Electric Products, Inc. for their cooperation in overcoming many difficult problems in vacuum-tube technic. Finally, he owes a great deal to the constant cooperation of his associates, particularly Wm. R. Campbell, Samuel Levy, and A. E. McPherson.

\section{ppendix}

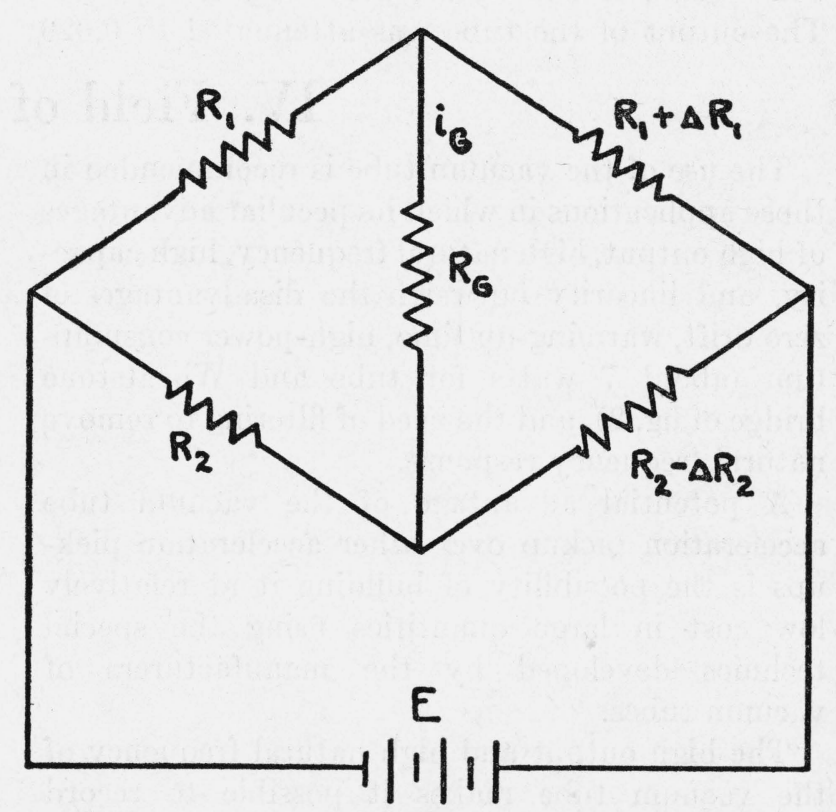

FIGURE 9.-Wheatstone bridge with small unbalance in two arms.

3 William H. Timbie and Vannevar Bush, Principles of electrical engineering (John Wiley \& Sons, Inc., New York, N. Y., 1930). 